\title{
Task Shifting: The Use of Laypersons for Acquisition of Vital Signs Data for Clinical Decision Making in the Emergency Room Following Traumatic Injury
}

\author{
Bryce E. Haac ${ }^{1} \cdot$ Jared R. Gallaher $^{2,3}$ - Charles Mabedi ${ }^{2} \cdot$ Andrew J. Geyer $^{4}$. \\ Anthony G. Charles ${ }^{1,2,3}$
}

Published online: 18 July 2017

\begin{abstract}
Importance In resource-limited settings, identification of successful and sustainable task-shifting interventions is important for improving care.

Objective To determine whether the training of lay people to take vital signs as trauma clerks is an effective and sustainable method to increase availability of vital signs in the initial evaluation of trauma patients.

Design We conducted a quasi-experimental study of patients presenting with traumatic injury pre- and postintervention.

Setting The study was conducted at Kamuzu Central Hospital, a tertiary care referral hospital, in Lilongwe, Malawi. Participants All adult (age $\geq 18$ years) trauma patients presenting to emergency department over a six-month period from January to June prior to intervention (2011), immediately post-intervention (2012), 1 year post-intervention (2013) and 2 years post-intervention (2014).

Intervention Lay people were trained to take and record vital signs.

Main outcomes and measures The number of patients with recorded vital signs pre- and post-intervention and sustainability of the intervention as determined by time-series analysis.

Results Availability of vital signs on initial evaluation of trauma patients increased significantly post-intervention. The percentage of patients with at least one vital sign recorded increased from 23.5 to $92.1 \%$, and the percentage of patients with all vital signs recorded increased from 4.1 to $91.4 \%$. Availability of Glasgow Coma Scale also increased from 40.3 to $88.6 \%$. Increased documentation of vital signs continued at 1 year and 2 years post-intervention. However, the percentage of documented vital signs did decrease slightly after the US-trained medical student and surgeon who trained the trauma clerks were no longer available in country, except for Glasgow Coma Scale. Patients who died during emergency department evaluation were significantly less likely to have vital signs recorded. Conclusions and relevance The training of lay people to collect vital signs and Glasgow Coma Scale is an effective and sustainable method of task shifting in a resource-limited setting.
\end{abstract}

Anthony G. Charles

anthchar@med.unc.edu

UNC Project, Lilongwe, Malawi

2 Department of Surgery, Kamuzu Central Hospital, Lilongwe, Malawi
3 Department of Surgery, UNC School of Medicine, Gillings School of Global Public Health, University of North Carolina at Chapel Hill, 4008 Burnett Womack Building, CB 7228, Chapel Hill, NC CB 7228, USA

4 Department of Mathematics and Statistics, Air Force Institute of Technology, Dayton, OH, USA 


\section{Introduction}

Vital signs (VS) have been shown to be useful in predicting mortality in critically ill patients and are essential for clinical decision making during the initial management of trauma patients [1-3]. In resource-limited settings, however, the critical dearth of healthcare personnel and lack of monitoring technology can result in the lack of routine acquisition of basic vital signs data in many patients $[4,5]$.

Sub-Saharan Africa, in particular, suffers from personnel and resource shortages that create significant obstacles in providing quality patient care [6-11]. This lack of resources has made it challenging for these countries to achieve health-related millennium development and sustainability goals. The modest interventions to increase the number of trained healthcare personnel cannot currently meet the healthcare demand. Malawi is one of the countries most effected by this crisis with a physician-to-population ratio of one per 50,000 and a critical nurse shortage with only 3.4 nurses per 10,000 population [7, 12].

Task shifting to non-physician healthcare workers has been shown to be an affordable and effective way to improve health care delivery in places with critical staff shortages [5, 13-20]. Task shifting is the process of transferring responsibility for designated tasks from one cadre of healthcare workers to another less specialized cadre in order to improve access to care and/or health care delivery without sacrificing quality. The WHO has recommended task shifting as a way to make more efficient use of the current human resources in the healthcare setting $[18,20]$. Studies have found that task shifting improves access to care for both communicable and non-communicable diseases without decreasing quality of care [5, 13-20]. In fact, task shifting has been associated with improved outcomes in a wide array of medical conditions including the treatment of HIV, hypertension, diabetes, mental health and surgical diseases including acute illness and injury. Given the worldwide surgical workforce shortage, task shifting is becoming increasingly common in surgical care $[21,22]$.

In the emergency department at Kamuzu Central Hospital (KCH) in Lilongwe, Malawi, many trauma patients are treated without any VS due to a lack of staff and equipment. A prior study from our institution revealed that task shifting the duty of VS measurement to lay people trained as vital sign assistants' was an effective way to increase the number of VS assessments in pediatric inpatient triage in our hospital [5]. We therefore sought to determine whether the training of lay people to take VS is an effective and sustainable method to increase availability of VS for clinical decision making in the initial evaluation of adult trauma patients. We hypothesized that task shifting the acquisition of VS from hospital nurses to lay people with at least a high school education would increase the number of patients with documented VS on presentation to the emergency department.

\section{Materials and methods}

\section{Study setting, design and population}

$\mathrm{KCH}$, located in the capital city of Lilongwe, is one of the Malawi's largest tertiary care hospitals with a busy trauma center serving a catchment of 800,000 from Lilongwe and referrals from Malawi's central region (pop. 5 million). The hospital has five surgical wards, including a dedicated burn center, and 235 adult surgical beds. On average, approximately 15,500 trauma patients present to the hospital's emergency department each year, and approximately $17.5 \%$ are admitted for their injuries. The hospital suffers from critical staff shortages with a patient-to-nurse ratio often exceeding 100:1 [5]. The number of clinical staff in the emergency department at a given time generally consists of two physicians (one medical officer and one intern) and two nurses. This low provider-to-patient ratio makes obtaining VS on all patients who present with trauma difficult, and as a result, many patients are treated, admitted and even sometimes discharged without a documented set of VS.

We conducted a quasi-experimental study examining VS collection in patients presenting to $\mathrm{KCH}$ with traumatic injury. We collected data on patients presenting over the same six-month period (January through June) pre-intervention in 2011, post-intervention (2012), 1 year post-intervention (2013) and 2 years post-intervention (2014) to eliminate potential for seasonal and annual variability. The majority of patients presenting with trauma over these time frames were male $(76.6 \%)$. The number of patients with recorded VS on presentation to the emergency department was compared over the aforementioned time frames in order to evaluate for immediate improvement in VS availability and sustainability.

\section{Intervention}

Preexisting trauma registry clerks were trained on VS collection during an initial 3-h training session including learning to collect manual blood pressure (BP), heart rate (HR) and respiratory rate (RR) and to calculate a Glasgow Coma Scale (GCS) in October 2011. During this session, clerks were taught the importance of VS including GCS, the range of normal VS and GCS for adult patients and how to obtain VS and to determine a patient's GCS. The clerks 
then practiced taking VS on each other and on patients waiting to be seen in the emergency department. They were also given a take-home test to practice determining the GCS. Test results were reviewed with each clerk individually if any errors were noted and all VS measurements during training were verified by a US-trained medical student and surgical resident who conducted the training session.

The clerks were instructed to collect VS on patients both on presentation to the emergency department and again within the first $36 \mathrm{~h}$ of admission. After the initial training session, a US-trained medical student or surgery resident rounded with the trauma clerks daily on the wards for 2 weeks and verified all manually taken VS and GCS determination at which point the measurements taken by the clerks corresponded to those taken by the resident or student. The medical student or resident continued to spot-check VS including rechecking any abnormal VS for an additional 2-week period and on an as needed basis thereafter. The clerks were given a chart with normal VS ranges and GCS calculation chart with guidelines for contacting a nurse or clinician if an abnormal VS or GCS was found. The same US-trained medical student and general surgery resident who had conducted the training remained available to help with any issues during the 6-month period immediately postintervention but were no longer in country at the 1-year and 2-year post-intervention time frames.

Clerks were then provided with and trained to use two automatic OmronBP760 7 Series Upper Arm Blood Pressure Monitor machines to allow for faster BP and HR measurement, one to be used in the emergency department and the other to be used on the wards. If the machines failed, clerks were instructed to revert to manual VS measurements. Clerks continued to collect respiratory rate manually.

Trauma registry clerks are lay people who had completed secondary school or higher and were able to read and write in both English and Chichewa, the local language. They are responsible for recording data for all trauma patients presenting to the hospital. $\mathrm{KCH}$ has ten trauma clerks who cover the emergency department $24 \mathrm{~h}$ per day, 7 days per week. One of the clerks sits at the intake desk at all times to check patients in, record their data for the trauma registry and direct patients to the appropriate patient care location based on the reason for seeking care. During the day, additional clerks round on admitted trauma patients to collect data on patients' clinical courses and enter data into the trauma registry.

\section{Data collection and analysis}

VS are one of the variables that the clerks are responsible for recording in the trauma registry if available. Prior to the intervention, nurses and physicians were responsible for taking vital signs. After the intervention, the trauma clerks took and recorded vital signs on patients independently and recorded them for the physician evaluation. Data were collected prospectively for all patients presenting to $\mathrm{KCH}$ with trauma and entered in the trauma registry. Demographic data and preliminary intervention effects were analyzed by comparing percentage of patients with recorded VS at each time point initially using a Chi-squared test.

Time-series analyses were then conducted using the $\mathrm{R}$ programming language in the MASS package to further delineate the effect of the intervention over time and to control for potential confounding variables [23]. Patients who were dead on arrival to the emergency department were excluded from analysis. Analysis of variance (ANOVA) was used to identify factors that had a significant effect on each outcome variable (HR, SBP, RR, GCS and if patients had all vital signs taken). A visual basic for applications script in MS Excel was used to count combinations of input variables and the number of times the vital sign of interest was recorded for a given combination of input variables during each 6-month period. Vital sign counts were used to generate negative binomial regressions with the count of each vital sign not taken as the output variable. The total count of each input variable combination was used as an offset in the regression to remove bias in the models due to differing total number of patients seen between the time periods. Input variables were checked for significance in the resulting negative binomial model, and counts were recalculated with the insignificant variables removed to create a negative binomial model with only significant input and minimal Akaike information criterion (AIC).

Resulting models were checked to ensure assumptions of the negative binomial model held. Likelihood ratio tests for the model parameters and the log likelihood test were performed to ensure the negative binomial was a more appropriate model than the related Poisson model. The negative binomial model was determined to be the best-fit model to use for analysis, as the conditional means did not appear to equal the conditional variances in all models.

\section{Ethics}

Both the Malawi National Health Science Research Council and the University of North Carolina IRB committees approved this study.

\section{Results}

Percentage of trauma patients with recorded VS during initial evaluation increased substantially post-intervention (Table 1; Fig. 1). During the 6-month pre-intervention 
period, from January to June $2011,23.5 \%$ of patients had at least one VS documented (HR, BP or RR) on presentation $(n=4212)$. During the immediate post-intervention 6-month period, the number of patients with at least one documented VS increased to $92.1 \%(n=4835, p<0.01)$. Compared to immediately post-intervention, at one-year post-intervention $82 \%$ of patients $(n=4631, p<0.01)$ and at two years post-intervention $84 \% \quad(n=4868$, $p<0.01$ ) of patients had at least one documented VS. The percentage of patients with all VS (HR, RR and BP) documented on presentation increased from $4.1 \%$ pre-intervention to $91.4 \%$ post-intervention $(p<0.01)$. This decreased to 81.2 and $83.7 \%$ of patients with all VS documented at 1 year post- and 2 years post-intervention, respectively $(p<0.01)$.

The percentage of patients with documented HR on presentation increased from 21.7 to $92 \%$ in the pre- intervention and immediate post-intervention periods, respectively $(p<0.01)$. Percentage of patients with documented HR remained high but was lower than immediately post-intervention at 1 year post-intervention $(82 \%$, $p<0.01)$ and at 2 years post-intervention $(83.8 \%$, $p<0.01)$. The percentage of patients with documented BP changed from $23.3 \%$ pre-intervention to $92 \%$ immediately post-intervention $(p<0.01)$. There was also a decrease in percentage of patients with a documented BP immediately post-intervention to $82 \% \quad 1$ year post-intervention $(p<0.01)$ and $83.8 \% 2$ years post-intervention $(p<0.01)$. The percentage of patients with documented RR improved from $5.3 \%$ pre-intervention to $91.6 \%$ immediately postintervention $(p<0.01)$, but the percentage again decreased to $81.3 \% 1$ year post-intervention $(p<0.01)$ and $84 \%$ 2 years post-intervention $(p<0.01)$ compared to immediately post-intervention. The percentage of patients with

Table 1 Percentage of patients with documented vital signs and GCS pre-intervention versus post-intervention over time

\begin{tabular}{llccrrr}
\hline Time frame & \% with one VS & \% with all VS & \% HR & \% BP & $\%$ RR & $\%$ GCS \\
\hline Pre-intervention & 23.5 & 4.1 & 21.7 & 23.3 & 5.3 & 40.3 \\
Post-intervention & 92.1 & 91.4 & 92.0 & 92.0 & 81.6 \\
1 year post-intervention & 82.0 & 81.2 & 82.0 & 82.0 & 81.3 \\
2 years post-intervention & 84.0 & 83.7 & 83.8 & 83.8 & 84.0 \\
\hline
\end{tabular}

Fig. 1 Change in percentage of documented vital signs over time. Percentage of all vital signs and GCS measurements available for clinical decision making during initial evaluation of trauma patients increased after the training of lay people to collect vital signs

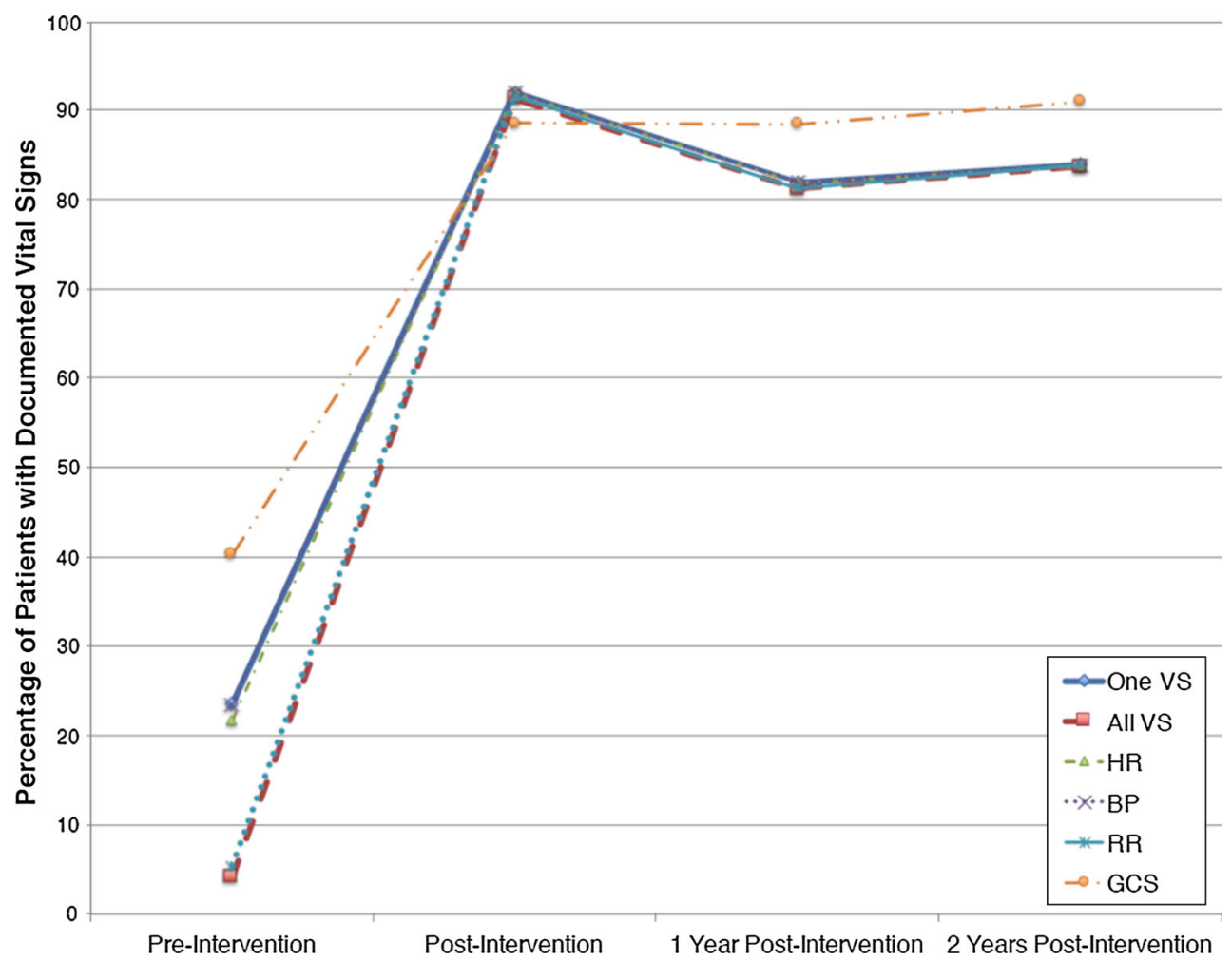


initial GCS documented increased from $40.3 \%$ pre-intervention to $88.6 \%$ post-intervention $(p<0.01)$ and remained at $88.5 \% 1$ year post-intervention $(p=0.91)$ and improved further to $91 \% \quad 2$ years post-intervention $(p<0.01)$.

In generating a negative binomial model for time-series analysis, the only factors that significantly affected whether a patient did not have vital signs recorded were time period of patient presentation, death in the emergency department (disposition) and the interaction between the above two factors $(p<0.0001$ for all VS and GCS, Table 2; Fig. 2). In time-series analysis, using the model to calculate relative risk of having VS taken post-intervention in comparison with patients prior to the intervention who survived in the emergency department, rates of vital signs being taken increased significantly for all patient dispositions in the year immediately following the intervention. However, rates 1 year and 2 years post-intervention decreased slightly while still remaining significantly higher than pre-intervention rates. Rates remained higher for all post-intervention years relative to the pre-intervention period for both patient dispositions when compared to a patient with the same disposition prior to the intervention. The intra-year incident rate ratio between patients who lived and those who died in the emergency department shows a trend where patients who eventually died in the emergency department have a much lower average rate of initial vital signs being taken than those who lived in the same year.

\section{Discussion}

Task shifting is a process of delegation whereby tasks are moved, where appropriate, to less specialized health workers. By reorganizing the workforce in this way, task shifting can make more efficient use of the human resources currently available [24]. Many countries, especially in sub-Saharan Africa, have adopted task shifting as an inevitable approach to alleviate human resource constraints. With fewer medical doctors at health institutions, nurses must carry out their own tasks as well as those normally performed by doctors. However, while doctors have managed to shift certain tasks to nurses so as to reduce their own workloads, nurses have shifted only a few tasks to others [25].

Based on our study results, task shifting the acquisition of VS from nurses and physicians to lay people with at least a secondary school education is a feasible, effective and sustainable intervention in a resource-limited setting. Availability of all VS and GCS for clinical decision making in initial trauma patient evaluation increased after the training intervention. Educating our trauma clerks on VS obtainment and GCS determination led to an over 22-fold increase in the number of patients with all VS, including $\mathrm{HR}$, BP and RR, documented on presentation and an almost fourfold increase in the number of patients with at least one of those VS documented. The largest improvement was in the number of patients with a documented respiratory rate. When controlled for patient disposition,

Table 2 Incident rate ratio of vital signs collected post-intervention compared to pre-intervention patients who survived beyond emergency department evaluation

\begin{tabular}{|c|c|c|c|c|c|c|c|c|c|}
\hline \multirow[t]{2}{*}{ Vital sign taken } & \multirow{2}{*}{$\begin{array}{l}\text { 6-month time frame } \\
\text { Outcome }\end{array}$} & \multicolumn{2}{|c|}{$\begin{array}{l}\text { Pre-intervention } \\
\text { (2011) }\end{array}$} & \multicolumn{2}{|c|}{$\begin{array}{l}\text { Immediately post- } \\
\text { intervention (2012) }\end{array}$} & \multicolumn{2}{|c|}{$\begin{array}{l}1 \text { year post- } \\
\text { intervention }(2013)\end{array}$} & \multicolumn{2}{|c|}{$\begin{array}{l}2 \text { years post- } \\
\text { intervention }(2014)\end{array}$} \\
\hline & & Lived & Died & Lived & Died & Lived & Died & Lived & Died \\
\hline \multirow[t]{2}{*}{ HR } & Lived & 1.0000 & 0.9497 & 11.5811 & 1.3370 & 4.7721 & 1.0091 & 5.0571 & 0.9696 \\
\hline & Died & 1.0530 & 1.0000 & 12.1946 & 1.4078 & 5.0249 & 1.0626 & 5.3250 & 1.0209 \\
\hline \multirow[t]{2}{*}{ RR } & Lived & 1.0000 & 1.1356 & 13.7545 & 1.6573 & 5.6613 & 1.2533 & 6.3611 & 1.1920 \\
\hline & Died & 0.8806 & 1.0000 & 12.1123 & 1.4594 & 4.9853 & 1.1036 & 5.6016 & 1.0497 \\
\hline \multirow[t]{2}{*}{ BP } & Lived & 1.0000 & 1.0131 & 11.3997 & 1.3173 & 4.6812 & 0.9939 & 4.9857 & 0.9450 \\
\hline & Died & 0.9870 & 1.0000 & 11.2521 & 1.3002 & 4.6206 & 0.9810 & 4.9212 & 0.9327 \\
\hline \multirow[t]{2}{*}{ All Vitals } & Lived & 1.0000 & 1.0677 & 13.5162 & 1.6698 & 5.6927 & 1.2635 & 6.3020 & 1.2018 \\
\hline & Died & 0.9366 & 1.0000 & 12.6595 & 1.5639 & 5.3319 & 1.1834 & 5.9025 & 1.1257 \\
\hline \multirow[t]{2}{*}{ GCS } & Lived & 1.0000 & 0.9095 & 5.6875 & 1.0753 & 5.9310 & 1.2216 & 6.4078 & 2.5198 \\
\hline & Died & 1.0995 & 1.0000 & 6.2534 & 1.1822 & 6.5211 & 1.3431 & 7.0453 & 2.7705 \\
\hline
\end{tabular}

Rate ratios were calculated from the negative binomial model generated in time-series analysis, and outcomes are denoted as column representing outcome in time frame being compared to baseline over row representing pre-intervention baseline outcome. Of note, there is a dramatic increase in availability of all VS and GCS immediately post-intervention with a slight but significant decrease in availability 1 year and 2 years post-intervention compared to immediately post-intervention $(p<0.0001)$. However, VS and GCS availability remains significantly increased compared to baseline at these time frames 

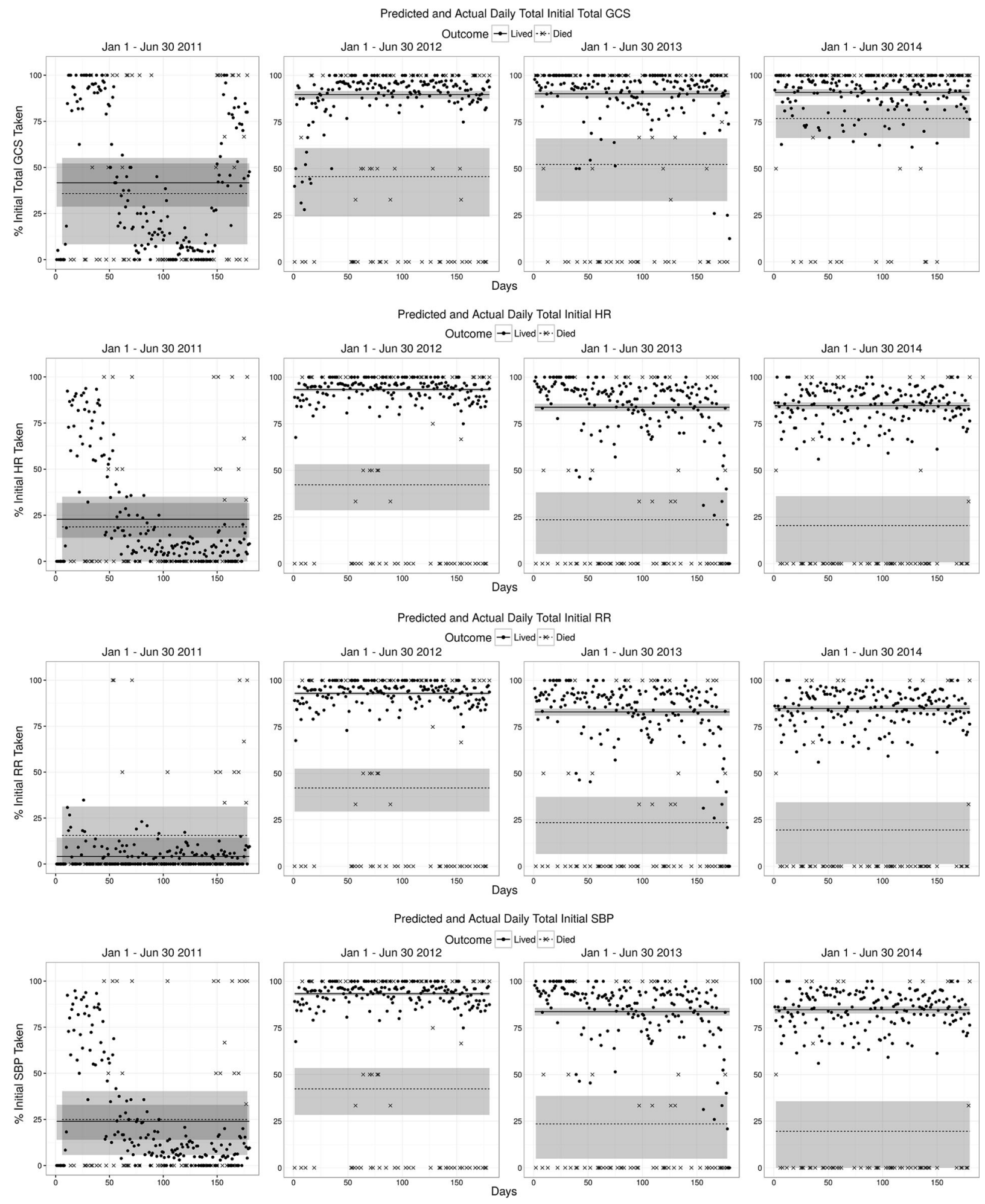

Fig. 2 Time-series analysis graphs of the effect of the intervention on vital signs availability during each time frame separating patients by emergency department disposition. Each graph is a representation of the percentage of patients with the specified vital sign taken on presentation to the ED by time frame day. Xs represents vital sign availability rates for patients who died during emergency department evaluation, and the dots represent rates for patients who survived beyond emergency department evaluation. The solid lines represent the average rate of VS/GCS availability for the specified time frame for patients who lived, and the dotted line represents the average rate for patients who died. The shaded area for each line represents the 95\% confidence interval with intervals that do not overlap between time frames being statistically significant 


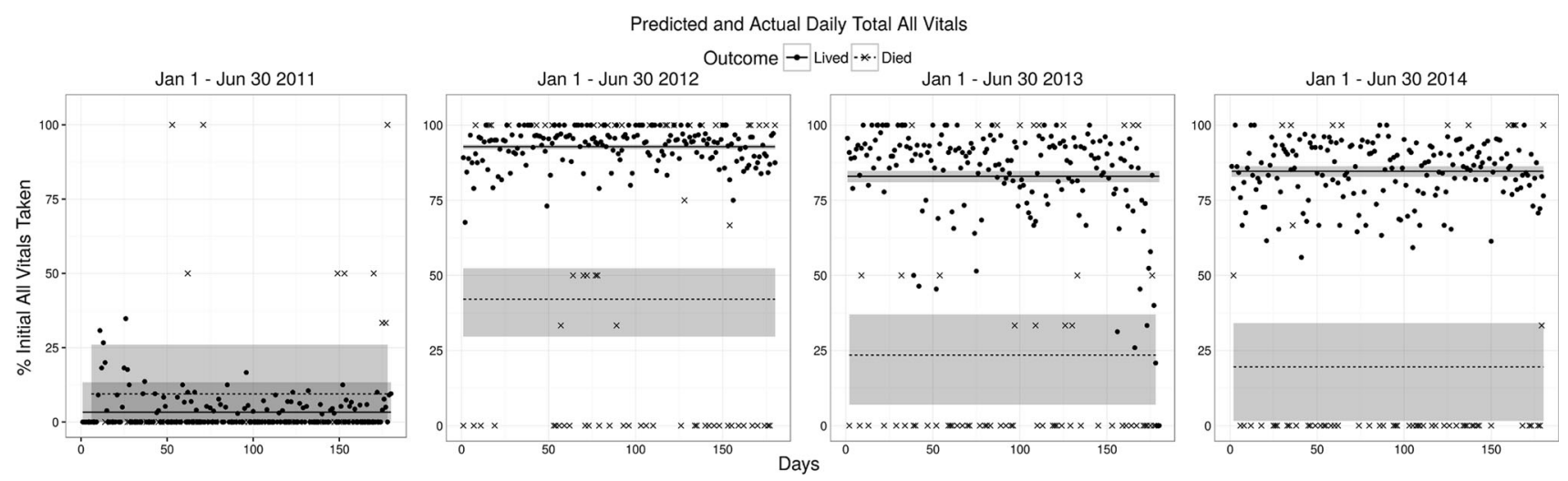

Fig. 2 continued

the rate of vital sign availability remained significantly higher, increasing over tenfold for all VS immediately post-intervention and over fivefold for GCS.

This increase in availability of VS was maintained with only a slight decrease in percentage of VS documented at 1 year and 2 years post-intervention once the US-trained medical student and general surgery resident that trained the clerks were no longer in country. There was no turnover in trauma clerks during the study period so this slight decline may be secondary to a decrease in supervision of the trauma clerks or due to patient presentation patterns. For example, if multiple patients present simultaneously, it is less likely that all patients will have documented VS compared to when the same number of patients present sequentially. Nevertheless, the percentage of patients with documented VS remained significantly higher in the postintervention period.

The trained trauma clerks were also able to determine and calculate the GCS resulting in over twice the number of patients having a documented GCS after the intervention compared to before the intervention. In fact, the percentage of patients with a documented GCS continued to increase significantly even after the US-trained medical student and general surgery resident that trained the clerks had left the country.

Patients who died during emergency department evaluation were less likely to have their vital signs taken, a trend that continued post-intervention. Reasons for this difference are likely multifactorial and, due to limitations of our study design, we are unable to speculate as to why this difference occurred. Future interventions should focus on increasing VS availability for these patients.

Task shifting must be implemented within systems that contain adequate oversight to protect both health workers and the patients. In developed countries, like the USA, task shifting the acquisition of vital signs to non-nurses is commonplace in surgical settings and serves as a strategy for providing health care in a cost-conscious manner. The success of this intervention has several implications for improvement of trauma care in resource-limited settings with minimal costs. First, the care burden on nurses and physicians in the emergency department is eased. Second, physicians are provided with valuable clinical data to inform their management plan that may not have otherwise been available. Third, patients may be triaged differently based on this new information resulting in fast tracking of patients with more serious injuries that may have otherwise been missed. Lastly, availability of VS information in trauma registries can improve the quality of retrospective registry reviews aimed at improving patient care.

Unfortunately, still several barriers exist that in the implementation of task shifting. Such barriers include: professional protectionism, where existing healthcare providers feel that their many years of training mean that not just anyone can do their work and consequently worry that their profession is being invaded by laypersons. Also, while the regulatory environment in some countries is permissive of task shifting, the cadre of laypersons may not have the necessary legal protection for additional tasks in the event of poor outcomes.

Limitations of this study include the inability to quantitatively describe the correlation between physician obtained vital signs and those measured by the data clerks. However, during the training, all of the clerks were qualitatively cleared by their trainers. Additionally, although we are able to measure the frequency of vital signs measurement at one and two years post-intervention, we did not measure the quality of the data recording because trainers were not available. Finally, this study was conducted as a quasi-experimental study so it is not possible to compare the acquisition of vital signs to a control group that did not have the intervention. As such, there could be other factors contributing to the improvement in VS acquisition over time that were not controlled for and we are unable to 
evaluate the effect of this improvement on patient outcome. However, the time-series analysis methods allow us to control for potential known confounding factors and to compare patients with similar characteristics clinical.

\section{Conclusion}

Task shifting has been embraced and accepted as a way to provide health care at reduced cost in high-income countries with abundant personnel and resources. The burden of surgical diseases in resource-poor countries is high and will remain insurmountable in the absence of task shifting as it offers high-quality, cost-effective care to more patients than a physician and nurse-centered model. Further studies are needed to determine the overall impact of this intervention on patient care and outcomes.

Acknowledgements Bryce Haac was supported by a Doris Duke International Clinical Research Fellowship.

\section{References}

1. Asiimwe SB, Abdallah A, Ssekitoleko R (2015) A simple prognostic index based on admission vital signs data among patients with sepsis in a resource-limited setting. Crit Care 16(19):86

2. Kause J, Smith G, Prytherch D, Parr M, Flabouris A, Hillman K, Intensive Care Society (UK), Australian and New Zealand Intensive Care Society Clinical Trials Group (2004) A comparison of antecedents to cardiac arrests, deaths and emergency intensive care admissions in Australia and New Zealand, and the United Kingdom-the ACADEMIA study. Resuscitation 62(3):275-282

3. Holcomb JB, Salinas J, McManus JM, Miller CC, Cooke WH, Convertino VA (2005) Manual vital signs reliably predict need for life-saving interventions in trauma patients. J Trauma 59(4):821-828 (discussion 828-9)

4. Asiimwe SB, Okello S, Moore CC (2014) Frequency of vital signs monitoring and its association with mortality among adults with severe sepsis admitted to a general medical ward in Uganda. PLoS ONE 9(2):e89879

5. Olson D, Preidis GA, Milazi R, Spinler JK, Lufesi N, Mwansambo C, Hosseinipour MC, McCollum ED (2013) Task shifting an inpatient triage, assessment and treatment programme improves the quality of care for hospitalised Malawian children. Trop Med Int Health 18(7):879-886

6. Chankova S, Muchiri S, Kombe G (2009) Health workforce attrition in the public sector in Kenya: a look at the reasons. Hum Res Health 7:58

7. Mills EJ, Schabas WA, Volmink J, Walker R, Ford N (2008) Should active recruitment of health workers from sub-Saharan Africa be viewed as a crime? Lancet 371(9613):685-688

8. WHO (World Health Organization) (2006) The world health report 2006: working together for health. WHO, Geneva, p 2006
9. Liese B, Dussault G (2004) The state of the health workforce in sub-Saharan Africa: evidence of crisis and analysis of contributing factors. In: Africa region human development working paper 32804, World Bank, Washington, DC

10. Smith MK, Henderson-Andrade N (2006) Facing the health worker crisis in developing countries: a call for global solidarity. Bull World Health Organ 84(6):426-427

11. Anand S, Barnighausen T (2004) Human resources and health outcomes: cross-country econometric study. Lancet 364(9445): $1603-1609$

12. The Henry J Kaiser Family Foundation (2016) Global health facts: nurses and midwives (per 10,000 population). http://kff.org/ global-indicator/nurses-and-midwives/

13. Ogedegbe G, Gyamfi J, Plange-Rhule J, Surkis A, Rosenthal DM, Airhihenbuwa C, Iwelunmor J, Cooper R (2014) Task shifting interventions for cardiovascular risk reduction in low-income and middle-income countries: a systematic review of randomised controlled trials. BMJ Open 4(10):e005983

14. Kredo T, Adeniyi FB, Bateganya M, Pienaar ED (2014) Task shifting from doctors to non-doctors for initiation and maintenance of antiretroviral therapy. Cochrane Database Syst Rev 7:CD007331

15. Joshi R, Alim M, Kengne AP, Jan S, Maulik PK, Peiris D, Patel AA (2014) Task shifting for non-communicable disease management in low and middle income countries-a systematic review. PLoS ONE 9(8):e103754

16. Mdege ND, Chindove S, Ali S (2013) The effectiveness and cost implications of task-shifting in the delivery of antiretroviral therapy to HIV-infected patients: a systematic review. Health Policy Plan 28(3):223-236

17. Chamberlain S, Stolz U, Dreifuss B, Nelson SW, Hammerstedt H, Andinda J, Maling S, Bisanzo M (2015) Mortality related to acute illness and injury in rural Uganda: task shifting to improve outcomes. PLoS ONE 10(4):e0122559

18. World Health Organization (WHO) (2006) Taking stock: task shifting to tackle health worker shortages, WHO health systems and services. www.who.int/healthsystems/task_shifting/TTR_ tacklepdf?ua $=1$

19. Dos Santos PF, Wainberg ML, Caldas-de-Almeida JM, Saraceno B, Mari Jde J (2016) Overview of the mental health system in Mozambique: addressing the treatment gap with a taskshifting strategy in primary care. Int J Ment Health Syst 10:1

20. WHO Guidelines Review Committee (2012) WHO recommendations: Optimizing health worker roles to improve access to key maternal and newborn health interventions through task shifting. WHO, Geneva

21. Daniels KM, Riesel JN, Meara JG (2015) The scale-up of the surgical workforce. Lancet 385(2):S41

22. Federspiel F, Mukhopadhyay S, Milsom P, Scott JW, Riesel JN, Meara JG (2015) Global surgical and anaesthetic task shifting: a systematic literature review and survey. Lancet 385(2):S46

23. Venables WN, Ripley BD (2002) Modern Applied Statistics with S, 4th edn. Springer, New York. ISBN 0-387-95457-0

24. WHO/PEPFAR/UNAIDS (2006) Treat, train, retain:task shifting: global recommendations and guidelines, WHO, Geneva. http:// data.unaids.org/pub/Manual/2007/ttr_taskshifting_en.pdf

25. Task Shifting to tackle health worker shortage (WHO). http:// www.who.int/healthsystems/task_shifting_booklet.pdf. Accessed 3 Nov 2016 